\title{
Designing Carbon Nanotube Membranes for Efficient Water Desalination
}

\author{
Ben Corry* \\ School of Biomedical, Biomolecular and Chemical Sciences, The University of Western Australia, \\ Crawley, Western Australia, 6009 Australia
}

Received: October 9, 2007; In Final Form: November 1, 2007

\begin{abstract}
The transport of water and ions through membranes formed from carbon nanotubes ranging in diameter from 6 to $11 \AA$ is studied using molecular dynamics simulations under hydrostatic pressure and equilibrium conditions. Membranes incorporating carbon nanotubes are found to be promising candidates for water desalination using reverse osmosis, and the size and uniformity of tubes that is required to achieve a desired salt rejection is determined. By calculating the potential of mean force for ion and water translocation, we show that ions face a large energy barrier and will not pass through the narrower tubes studied $((5,5)$ and $(6,6)$ "armchair" type tubes) but can pass through the wider $(7,7)$ and $(8,8)$ nanotubes. Water, however, faces no such impediment due to the formation of stable hydrogen bonds and crosses all of the tubes studied at very large rates. By measuring this conduction rate under a hydrostatic pressure difference, we show that membranes incorporating carbon nanotubes can, in principle, achieve a high degree of desalination at flow rates far in excess of existing membranes.
\end{abstract}

\section{Introduction}

One impact of global climate change has been the redistribution of rainfall that is leading to increasingly dry conditions in many areas. ${ }^{1}$ In Australia, for example, a drop in rainfall in most populated areas in conjunction with an increasing population in the urban centers has meant that water is becoming scarce for agricultural use and that all of the major cities are undergoing severe restrictions on water use. Desalination of seawater has long been utilized to produce fresh water suitable for human consumption and irrigation in areas of water scarcity, and its use is becoming more widespread across the globe. The most common method of desalination known as "reverse osmosis" involves the use of semipermeable membranes to filter out dissolved salts and fine solids. High pressure, and thus significant energy, is required to force water through the membranes. The cost of supplying this energy forms one of the major impediments to wide scale use of seawater desalination plants. One mechanism for alleviating these costs is to develop nanoporous semipermeable membranes containing continuous channels that can pass a greater volume of water at a given pressure than existing membranes while still blocking the passage of ions. A comparison with biological systems suggests that there is some hope in achieving this goal as there exist biological water pores, known as aquaporins, that are able to rapidly pass water and block ions. ${ }^{2-4}$

Carbon nanotubes (CNTs) have received considerable interest since their discovery in $1991^{5}$ due to their unique mechanical, optical, and electrical properties that make them amenable to a range of applications including catalysis, electronics, and molecular sensing. ${ }^{6-8}$ CNTs have also been demonstrated as a useful material for manufacturing electrodes for use in desalination of water using a flow through capacitor., ${ }^{9,10}$ Single, double, and multiwalled carbon nanotubes have been made of various sizes, and recent fabrication techniques have enabled

* To whom correspondence should be addressed. E-mail: ben.corry@ uwa.edu.au. the arrangement of aligned tubes of controllable sizes ${ }^{11-14}$ and within membranes ${ }^{15-18}$ which has opened the door to applications involving filtration. Measurements have shown selective gas transport can be obtained in $<2 \mathrm{~nm}$ diameter carbon nanotube membranes ${ }^{18}$ supporting previous simulation studies. ${ }^{19}$

Previous experimental ${ }^{18}$ and simulation studies ${ }^{20-22}$ have indicated that water can permeate through relatively narrow carbon nanotubes. Furthermore, flow rates have been found to be unexpectedly high and mostly independent of the length of the pores in contrast with predictions from macroscopic hydrodynamics. These results are surprising as it has often been thought that the nonpolar interior of narrow nanotubes would provide an unfavorable home for polar water molecules. Indeed, there is mounting evidence that a number of biological ion channels are able to block the passage of both water and ions in their closed state by providing a narrow, but not occluded, hydrophobically lined pore. Simulation studies on model pores, ${ }^{23,24}$ the so-called mechanosensitive channel of small conductance $^{25,26}$ and the nicotinic acetylcholine receptor ${ }^{27,28}$ indicate that there is a critical pore diameter of $\sim 7 \AA$, above which ions and water will pass but below which they will not. At this critical radius, however, it is possible that water may be able to permeate through these pores, whereas ions do not.

Reports of rapid water conduction through narrow $(6,6)$ carbon nanotubes, however, are particularly intriguing as these may be promising for forming semipermeable membranes that filter salt from water. ${ }^{21}$ The fast transport properties for both gas and water in nanotubes ${ }^{18}$ also supports the belief that filtration may be achieved without having to trade off against the transport rate. Previous molecular simulation studies have examined the formation of "water wires" through CNTs and proton conduction along these..$^{20,29-32}$ A number of studies have also examined the structure of water inside and near CNTs, finding that water tends to form very ordered structures inside and at the mouth of neutral and charged pores. ${ }^{21,33-36}$ As described above, there have been some studies that aimed to determine the conductance of water through CNTs under 


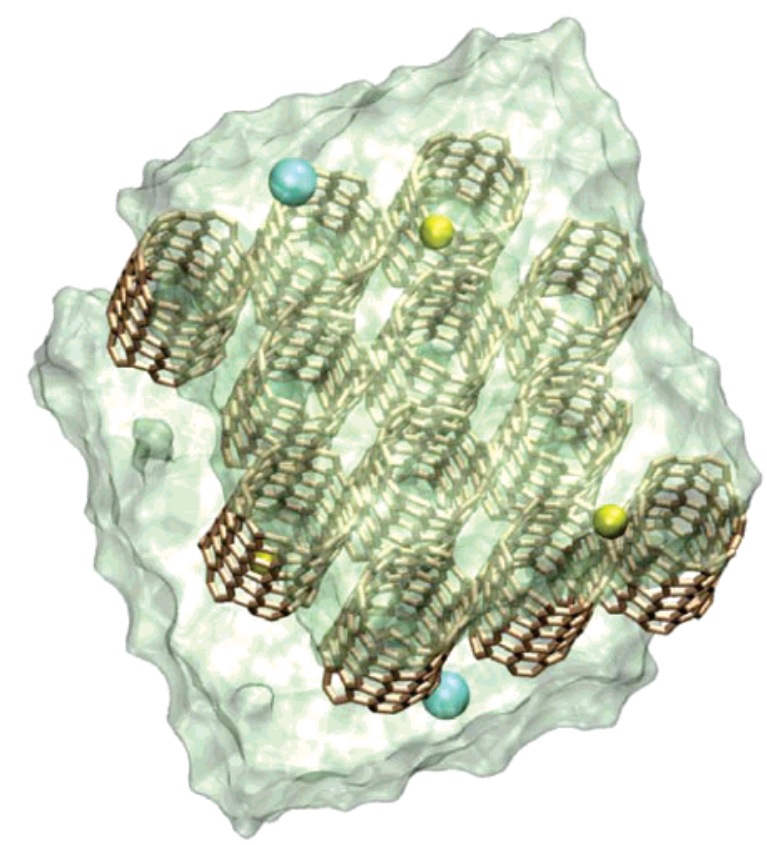

Figure 1. Simulation system. A CNT membrane is formed by hexagonally packing 12 nanotubes in a periodic cell. The full simulation system is formed by placing layers of water on either side of the membrane (light blue surface) and adding ions to the bulk solution.

osmotic $^{21}$ or hydrostatic pressures ${ }^{32}$ in neutral and charged $(6,6)$ nanotubes. Although there has been an investigation into the energy barrier presented to $\mathrm{Na}^{+}$in two differently sized CNTs $((6,6) \text { and }(10,10))^{37}$ and an examination of the hydration structure of ions in two narrow CNTs, ${ }^{38}$ there is yet to be a study that systematically quantifies the conductance of both water and ions in a range of differently sized CNTs to determine the likely salt rejection of each when used in reverse osmosis (rather than in flow through capacitors).

In this study the energetics of water and ion transport are examined in a range of differently sized carbon nanotubes such that their applicability for use in water desalination can be investigated. Using a total of $500 \mathrm{~ns}$ of molecular dynamics simulations, we determine the energy barriers to ion and water conductance in membranes formed from $(5,5),(6,6),(7,7)$, and $(8,8)$ "armchair" type carbon nanotubes and examine their conduction rate when a hydrostatic pressure difference is applied. The nature of molecular transport can be derived as well as the specific energetic contributions that assist or prevent conduction. Most importantly, this study predicts that efficient desalination should be possible using membranes incorporating carbon nanotubes and enables estimates to be made about the required uniformity in size that is required to produce water of sufficient purity for human consumption.

\section{Materials and Methods}

Carbon nanotube membranes were modeled following the technique of Zhu and Schulten ${ }^{32}$ in which 12 carbon nanotubes are hexagonally packed in a regular arrangement as shown in Figure 1. Periodic boundaries were employed to form a continuous 2-dimensional membrane that was solvated by $\sim 25$ $\AA$ thick layers of water (using the TIP3P force field) on either side with $\mathrm{Na}^{+}$and $\mathrm{Cl}^{-}$randomly placed to yield a net concentration of $250 \mathrm{mM} \mathrm{NaCl}$. Five separate simulation systems were constructed comprising $\sim 14 \AA$ long tubes of $(5,5)$, $(6,6),(7,7)$, and $(8,8)$ symmetry and longer $\sim 28 \AA(6,6)$ nanotubes. The diameters of the nanotubes as measured between the carbon centers as well as the effective internal diameter
TABLE 1: Differences in the Nonbonded Energies for an Ion (Upper Rows) or Water (Lower Rows) in the Bulk and in the Center of the Nanotube ${ }^{a}$

\begin{tabular}{lrrrrr}
\hline & \multicolumn{5}{c}{ energy change upon entering pore (kcal/mol) } \\
\cline { 2 - 6 } size & total & $\begin{array}{c}\text { neighboring } \\
\text { water }\end{array}$ & $\begin{array}{c}\text { pore water } \\
\text { (excl. neighbors) }\end{array}$ & nanotube & bulk \\
\hline \multicolumn{5}{c}{$\mathrm{Na}^{+}$} \\
$(5,5)$ & 12.06 & 10.05 & -1.29 & -2.21 & 5.51 \\
$(6,6)$ & 11.44 & 8.12 & -4.39 & -1.65 & 9.37 \\
$(7,7)$ & 3.69 & 4.45 & -4.67 & -0.96 & 4.87 \\
$(8,8)$ & -1.60 & 0.77 & -9.15 & -0.56 & 7.34 \\
& & \multicolumn{5}{c}{$\mathrm{H}_{2} \mathrm{O}$} & & \\
$(5,5)$ & -3.90 & -0.22 & -0.31 & -5.41 & 2.04 \\
$(6,6)$ & -3.57 & 0.01 & -0.02 & -5.16 & 1.60 \\
$(7,7)$ & -2.34 & 0.32 & 0.01 & -3.73 & 1.70 \\
$(8,8)$ & -1.63 & 1.16 & 0.03 & -2.91 & 0.09
\end{tabular}

${ }^{a}$ The total energy is decomposed into contributions arising from the neighboring water molecules, the single file of water in the pore, the van der Waals interactions with the carbon atoms (nanotube) and the remaining interactions with the bulk water.

TABLE 2: Water and Ion Conductance of Nanotubes under $208 \mathrm{MPa}$ Pressure at $250 \mathrm{mM}$ Ion Concentration ${ }^{a}$

\begin{tabular}{|c|c|c|c|c|c|}
\hline \multirow[b]{2}{*}{ size } & \multicolumn{2}{|c|}{ diameter $(\AA)$} & \multirow{2}{*}{$\begin{array}{l}\text { run length } \\
\text { (ns) }\end{array}$} & \multicolumn{2}{|c|}{ conductance pt pns } \\
\hline & $\mathrm{C}-\mathrm{C}$ & internal & & $\mathrm{H}_{2} \mathrm{O}$ & ions \\
\hline$(5,5)$ & 6.6 & 3.2 & 10.00 & $10.4 \pm 0.4$ & 0.0 \\
\hline$(6,6)$ & 8.1 & 4.7 & 20.00 & $23.3 \pm 0.3$ & 0.0 \\
\hline$(7,7)$ & 9.3 & 5.9 & 25.00 & $43.7 \pm 0.5$ & $0.007 \pm 0.005$ \\
\hline$(8,8)$ & 10.9 & 7.5 & 17.00 & $81.5 \pm 1.2$ & $0.137 \pm 0.025$ \\
\hline$(6,6)$ long & 8.1 & 4.7 & 10.00 & $23.4 \pm 0.5$ & 0.0 \\
\hline
\end{tabular}

${ }^{a}$ The conductance per tube per ns for water and ions are shown as well as the size of each pore measured between the center of the carbon atoms and the effective internal diameter of the pores.

assuming a carbon atom van der Waals radius of $1.7 \AA$ are indicated in Table 2. Following Hummer et al., ${ }^{20,21}$ all carbon atoms were taken to be neutral. Although recent studies suggest that the atoms at the very end of the tube are likely to have slightly more charge than those in the center, ${ }^{39}$ we expect this to have only a minor influence on the conduction and ion exclusion properties of the nanotubes. An examination of this may be worthwhile at a later date. Force constants were defined as those aromatic carbons (CA) in the CHARMM27 parameter set. Before collecting data, all systems were energy minimized and equilibrated for $0.5 \mathrm{~ns}$ under a constant pressure of $1 \mathrm{~atm}$ and temperature of $300 \mathrm{~K}$ during which time a harmonic constraint applied to the carbon atoms was gradually reduced from 1 to $0.1 \mathrm{kcal} / \mathrm{mol} / \AA^{2}$. Apart from the simulations conducted with pressure differences discussed below, harmonic constraints of $0.1 \mathrm{kcal} / \mathrm{mol} / \AA^{2}$ were applied to all of the carbon atoms during data collection to keep the membrane roughly in place (although the CNTs remained well packed in the membrane during a $5 \mathrm{~ns}$ simulation without any restraints on the carbon atoms).

Potentials of mean force (PMF) for $\mathrm{Na}^{+}$were determined using umbrella sampling ${ }^{40}$ in which a harmonic biasing potential $\left(K_{r} / 2\right)\left(r-r_{i}\right)^{2}+\left(K_{z} / 2\right)\left(z-z_{i}\right)^{2}$ was applied to a test ion, where $r$ and $z$ are the radial and axial coordinates of the ion defined from the center of one of the pores, $r_{i}$ and $z_{i}$ are target ion positions, and $K_{r}$ and $K_{z}$ are corresponding force constants. The target positions were moved from $z=14$ to $z=-1 \AA$ with $r$ $=0$ and from $z=14$ to $z=5 \AA$ with $r=5 \AA$ using force constants $K_{r}=0.1$ and $K_{z}=1.0 \mathrm{kcal} \mathrm{mol}^{-1} \AA^{2}$. Collective analysis of the data was made using the weighted histogram analysis method ${ }^{41,42}$ using the implementation of Grossfield. PMF for water was detetrmined using the average densities in equilibrium simulations using the fact that $\operatorname{PMF}(z, r)=-k T$ 
$\ln \left(\rho(z, r) / \rho_{0}\right)$ where $\rho$ is the atom density. In both cases the 2-dimensional PMF was renormalised to account for the size of the volume element $2 \pi r$. One-dimensional PMF were then obtained by integrating the renormalised probabilities of each ion position $P(z, r)$ in the $x-y$ plane up to $r=5 \AA$

$$
P M F(z)=-k T \ln \left(\int_{0}^{5} P(z, r) \mathrm{d} r\right)
$$

A method developed by Zhu et al. was employed to introduce a hydrostatic pressure difference in our simulations. ${ }^{43,44}$ Because of the periodicity of the system, the water forms layers sandwiched between adjacent nanotube membranes. Each water layer is divided into three sections, and a constant force, $f$, related to the desired pressure difference, $\Delta P$, is applied in the $-z$ direction to all water molecules in this middle section to create a pressure differential across the membrane using the fact that $\Delta P=n f / A$ where $n$ is the number of water molecules in the section to which forces are applied and $A$ is the cross sectional area of the membrane. ${ }^{43}$ In our case, the constant force was applied to water molecules greater than $13 \AA$ from either side of the membrane. To keep the membrane in place and avoid overall translation of the system, harmonic constraints of 0.8 $\mathrm{kcal} / \mathrm{mol} / \AA^{2}$ were applied to all of the carbon atoms. Simulations involving hydrostatic pressure differences were performed under constant volume and constant temperature $(300 \mathrm{~K})$ conditions using a langevin thermostat. In order to keep the pressure close to the specified value during simulations, an ion concentration of $250 \mathrm{mM}$ was used rather than the greater concentration of seawater to avoid large osmotic pressure differences arising during the simulations. Simulations were conducted with NAMD $^{45}$ with the CHARMM 27 force field ${ }^{46}$ with a 1 ps time step using a particle mesh ewald scheme for electrostatic calculations.

\section{Results and Discusssion}

Water in the Pores. Membranes were formed by hexagonally packing CNTs (Figure 1), and during equilibration, water was found to enter all four differently sized nanotubes. A single file chain of water is seen in the $(5,5)$ and $(6,6)$ tubes, whereas a double chain is seen in the $(7,7)$ tube and a quadruple chain seen in the $(8,8)$ pore as indicated in Figure 2A. Ions, on the other hand, did not enter any of the pores during this initial equilibration period. The entry of water was very rapid, with every pore filled within $0.1 \mathrm{~ns}$ in all except the $(5,5) \mathrm{CNT}$. Indeed, water never fills all of the available pores for this narrowest CNT, but rather, each pore in the membrane alternates between two hydration states, either fully occupied (as seen in Figure 2A) or completely empty, indicating the large energetic cost involved in fragmenting the hydrogen-bonded chain. ${ }^{20}$ The number of water molecules in one of the $(5,5)$ pores is illustrated in Figure 3A during a 14 ns equilibrium simulation and can be seen to fill and empty of water three times. The number of filled pores (out of a maximum of 12) at any time during the simulation is seen in Figure 3B, showing that after equilibration on average just over half of the pores are hydrated. Some caution should be taken when using these results to determine the most likely hydration state of the $(5,5)$ pore, as it has previously been shown that this is dependent upon the exact choice of LennardJones parameters used to model the van der Waals interactions between the water oxygen and carbon atoms. ${ }^{20}$

The average oxygen density in the central $7 \AA$ of the pores during $1.5 \mathrm{~ns}$ of simulation is plotted in Figure 2B. It can be seen that while the narrow dimension of the $(5,5)$ tube forces the water to reside in the center of the pore, the $(6,6)$ tube is
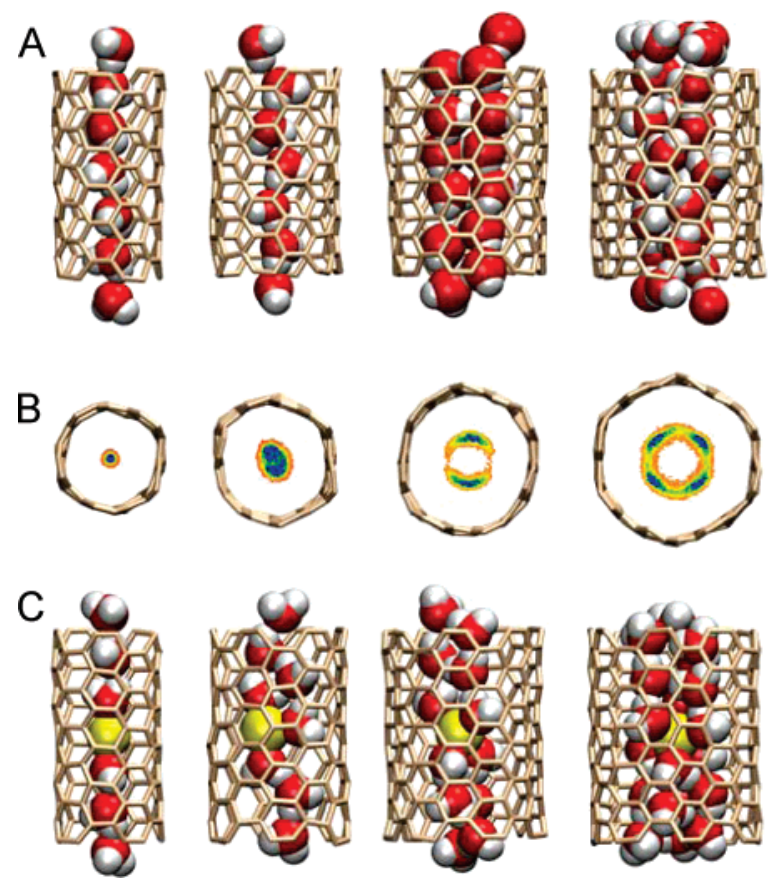

Figure 2. Water and ions in the nanotubes. (A) Snapshots from molecular dynamics simulations showing the configuration of water in each of the $(5,5),(6,6),(7,7)$, and $(8,8)$ CNTs are shown as viewed from the plane of the CNT membrane. (B) Top views of the nanotubes show the differing sizes of the tubes as well as the structure of water in the pores as evidenced from the density of oxygen in the central 7 $\AA$ of the pore. Density values range form low (orange) to high (blue). (C) Location and hydration structure of $\mathrm{Na}^{+}$ions that are pulled into the center of the pores.

wide enough for the molecules to have some degree of radial freedom. Water forms surprisingly ordered structures in both the $(7,7)$ and $(8,8)$ pores. In the former, hydrogen bonds form along the two chains of water molecules as well as between them to maintain a stable structure, whereas a similar picture emerges for the four water chains passing through the $(8,8)$ CNT. There is also a slight elongation of the $(7,7)$ CNT perpendicular to its axis to accommodate the two water chains as can be seen after careful inspection of Figure 2B. Highly ordered structures of water have been seen inside simulations of wider nanopores ${ }^{35}$ as well as in CNTs that are cooled under pressure. ${ }^{33}$ This structuring has been suggested to arise from the best match of the oxygen and carbon van der Waals interactions as well as the formation of optimal hydrogenbonding networks and has also been strongly linked to the transport properties of ions in the pore. ${ }^{37,47}$

PMF for Ions and Water. In order to calculate the likelihood of ions and water passing through the nanotubes, the potential of mean force as either an ion (Figure 4A) or water (Figure 4B) is brought from bulk $(z=14 \AA)$ into the center of each of the differently sized tubes $(z=0)$ is calculated (right to left in Figure 4). In the two narrowest pores, there is a very large free energy barrier that can be expected to prevent ion conduction arising predominantly at the entrance of the CNTs. In the wider tubes, this barrier is significantly smaller, suggesting that ions could pass through them.

There is no large free energy barrier to water conduction in any of the CNTs studied. In all but the narrowest pore, the largest energy barrier is less than $0.4 \mathrm{kcal} / \mathrm{mol}$. The small free energy barrier outside the mouth of the tube occurs due to the large van der Waals interactions of the oxygen and carbon atoms that create a structuring of molecules near the nanotube-bulk water interface that has been described previously. ${ }^{21}$ These 


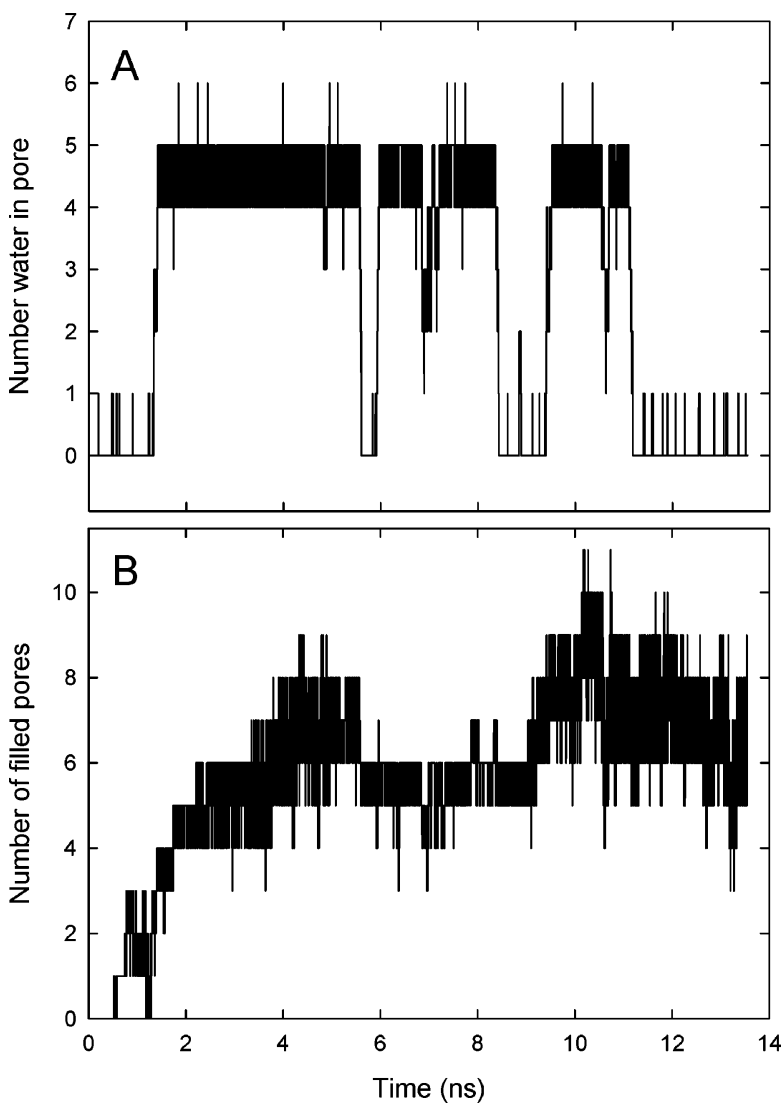

Figure 3. Water occupancy of the $(5,5)$ nanotube. (A) The number of water molecules inside one tube and (B) the number of tubes (out of a maximum of 12) containing more than three water molecules are plotted as a function of time.

PMFs suggest that there should be no significant impediment to water conduction through any of these nanotubes.

In Figure 4, panels C and D, the origins of the free energy barriers are examined. The narrower CNTs are not large enough to allow an ion to enter with its hydration shell intact and stripping the surrounding water comes at an energy cost that causes the barriers seen in Figure 4A. In the simulations, it is found that the $\mathrm{Na}^{+}$ion has a fairly constant coordination number a little under 6 in the bulk solution (determined by counting the number of oxygen atoms within $3.2 \AA$ ), but to enter the narrowest $(5,5) \mathrm{CNT}$, all but two of these must be removed so that the ion is in contact with just one water molecule on either side as illustrated in Figure 2C. In effect, the ion replaces one of the water molecules in the single file chain passing through the pore. Despite the $(6,6) \mathrm{CNT}$ only supporting a single file of water as illustrated in Figure 2A, it is roomy enough for an $\mathrm{Na}^{+}$to contact 3 water molecules inside the pore (Figure $2 \mathrm{C}$ ). In the $(7,7) \mathrm{CNT}$, only one or two water molecules must be removed from the solvation shell, whereas in the $(8,8)$ tube the ion can enter with its first solvation shell remaining intact.

To better understand the energetics of moving an ion into the nanotubes, we decompose its interactions with surrounding molecules in Table 1. The change in the nonbonded interaction energies of the ion upon moving to the center of the pore from the bulk are broken into 4 components: interactions with the immediate neighboring water molecules (i.e., the first solvation shell), the water inside the pore (excluding immediate neighbors), the van der Waals interactions with the carbon atoms forming the nanotubes, and the remaining bulk water. The difference in these interactions when the ion is in the center of the pore compared to when it is in the bulk shows that stripping water from the solvation shell of the ions has an energy cost of about $2.5 \mathrm{kcal} / \mathrm{mol}$ per water molecule (as calculated by dividing the energy contribution by the decrease in solvation number). There is also a similar cost involved in moving the ion from a region of bulk water to the lower dielectric region of the nanotube membrane. However, these costs are reduced by favorable van der Waals interactions with the narrow nanotubes and with an attraction from the water molecules in the pore whose aligned dipoles can create a significant force on the ion. This attraction to water in the pore is greatest in the large $(8,8)$ $\mathrm{CNT}$ as it contains the most water molecules and they remain well aligned, showing that such dipole alignment does not only occur for a single file of water. It should be noted that the total energy difference indicated in the table cannot be directly compared to the PMF as the results in the table do not include the energy to expel a water molecule to be replaced by the ion nor that relating to bond stretching or bending or the access resistance involved in moving a particle from bulk into a narrow pore.

Although an individual water molecule does not maintain a solvation shell in the way that an ion does, water is known to form a large number of hydrogen bonds with surrounding molecules in bulk solution. To enter the nanotubes, however, some of these bonds must be broken as illustrated in Figure 4D. Following the suggestion of ref 48, hydrogen bonds on a water molecule are counted for any hydrogen oxygen pair within $2.4 \AA$ regardless of the angle. In the wider pores, each water molecule can maintain close to the bulk number of hydrogen bonds as it enters the pore, as they form orderly hydrogenbonded networks as described above. The fact that water is restricted to a single file through the two narrowest CNTs, however, implies that each water molecule can only participate in two hydrogen bonds, one with the water on each side. Thus, one might expect that there would be a significant energy cost to move a water molecule into the pore as two hydrogen bonds must be broken. However, this energy cost must be small as seen in the PMF shown in Figure 4B.

To better understand why the removal of hydrogen bonds does not create a barrier to water permeation, we also decompose the interactions between the water molecule and its surroundings in Table 1. Surprisingly, there appears to be no significant cost in breaking the hydrogen bonds as required for water to enter the narrow pores as there is very little difference in the interaction energy with the neighboring water molecules in the bulk and in the pore. This can be understood by examining the energy of these interactions during dynamic simulations. In the bulk solution, these interactions with neighboring waters fluctuate significantly. Although it is possible for any given water molecule to have a greater favorable interaction with the surrounding water molecules in bulk, the increased freedom of the molecules means that the configurations that give very favorable interactions are compensated by less favorable ones. Inside the nanotubes, however, the interactions between the water molecules are much more stable as suggested by Hummer et al., ${ }^{20}$ with much smaller fluctuations seen in the size of the interactions, yielding a very similar average interaction energy. Previous studies have also noted that when hydrogen bonds between water molecules in CNTs do break, they can reform quickly as adjacent water molecules are forced to remain in the vicinity. ${ }^{36}$ A similar phenomena of long lasting hydrogen bonds has also been suggested in studies in an $(8,8)$ nanotube. ${ }^{22}$ It is also notable that there are very large van der Waals interactions between the water oxygen atoms and the carbon atoms. This leads to a larger favorable interaction between water and the 

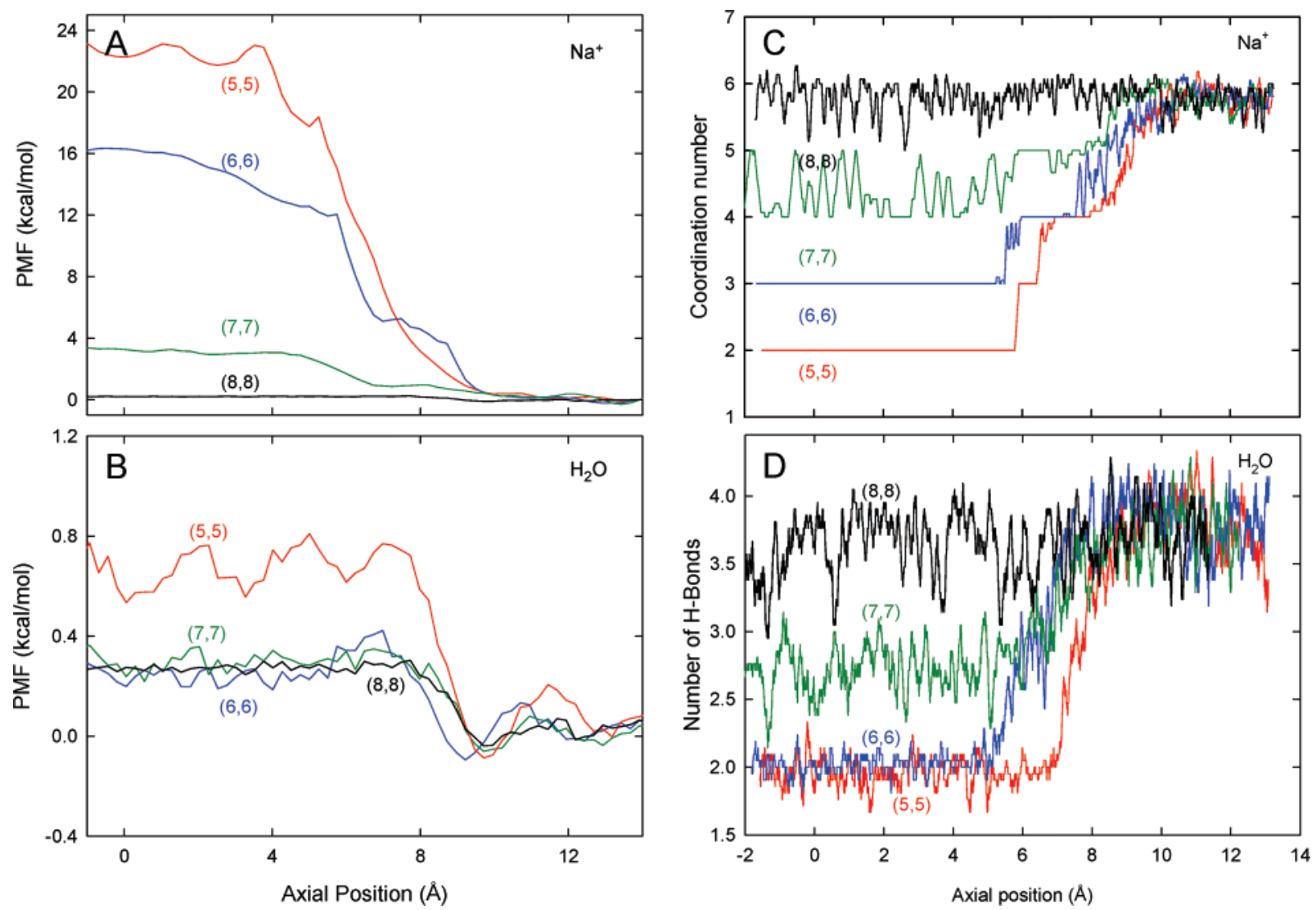

Figure 4. Potential of mean force and coordination numbers for ion and water permeation into a CNT membrane. The PMF is shown for pulling an ion (A) and water (B) into each of the $(5,5),(6,6),(7,7)$, and $(8,8)$ nanotubes. The ion or water is moved from the bulk solution $(z=14)$ into the center of the pore $(z=0)$ in each case. The coordination number of $\mathrm{Na}^{+}(\mathrm{C})$ and the number of hydrogen bonds formed with the water molecule (D) are indicated. The location of the nanotube is indicated by the gray background.

narrow tubes, than water with the wider tubes (first data column in Table 1). The strength of this interaction is highly dependent on the choice of Lennard Jones parameters used in the simulations, ${ }^{20}$ so some caution should be taken when interpreting this result. The interaction between water and the narrow tubes does significantly impede the conductance of water in the pores as described below.

To examine the effect of the length of the CNT on the PMF, simulations were conducted on a $(6,6)$ nanotube double the length $(\sim 28 \AA)$ of the tubes described above. The energy profiles for ion and water permeation are similar to that seen in the shorter tube (data not shown). Once inside the pore, the PMF is flat, indicating that there are no additional energy barriers for an ion to move through a long nanotube than a short one. This suggests that the center of the tubes are almost frictionless and we should expect the conductance of water through these CNTs to be largely independent of their length as suggested previously, ${ }^{21}$ a point that is proven below.

Simulations under Hydrostatic Pressure. The most important information for assessing the suitability of CNTs for desalination is a direct measure of the water and ion conductance through them. To determine this, a hydrostatic pressure difference was created across the CNT membranes and the number of water molecules and ions that pass across it during our simulations was counted. The transport of water through these pores is remarkably fast, in agreement with previous simulations in the $(6,6)$ nanotube. ${ }^{20,21} \mathrm{~A}$ similar phenomenon for gas transport has been ascribed to the inherent smoothness on the CNT walls. ${ }^{49}$ Water molecules move through the CNTs in a concerted motion as illustrated for the $(6,6)$ CNT under a 208
MPa hydrostatic pressure difference in Figure 5A. Here, the axial position $(z)$ of oxygen atoms in a tube are plotted over time, illustrating that, although water occasionally steps backward, a net flow of molecules through the tubes is observed and a continuous chain of water is always seen in the simulation. In Figure 5B, we show the relationship between the water conductance of a $(6,6)$ CNT membrane and the hydrostatic pressure difference, obtained without any ions in the simulation. The conductance is found to increase linearly over the range of pressures from 5 to $400 \mathrm{MPa}$. Although most reverse osmosis membranes operate under pressures of less than $10 \mathrm{MPa}$, we conduct the remainder of our simulations under a larger $\sim 200$ MPa pressure difference as this allows for a greater number of conduction events to be observed in our limited simulations time, and the results at this pressure can be easily extrapolated to lower pressures.

In Table 2 and Figure 5C, we show the conductance of water and ions (number of molecules crossing the membrane in a given time) measured in our simulations for the range of different sized CNTs studied with $250 \mathrm{mM} \mathrm{NaCl}$ solution. Water is seen to pass through all of the nanotubes, and not surprisingly, the conductance is greater in the wider tubes that the narrow ones. The conductance of the long $(6,6)$ tube is similar to that of the shorter tube, supporting our claim and previous results ${ }^{21}$ that the conductance is largely independent of tube length. It should be noted that this directed conductance of water molecules is measured under a driving force, in this case a hydrostatic pressure differential. In contrast, the conductance of water molecules by chance wanderings in either direction through nanotubes in equilibrium conditions would be expected to decay 

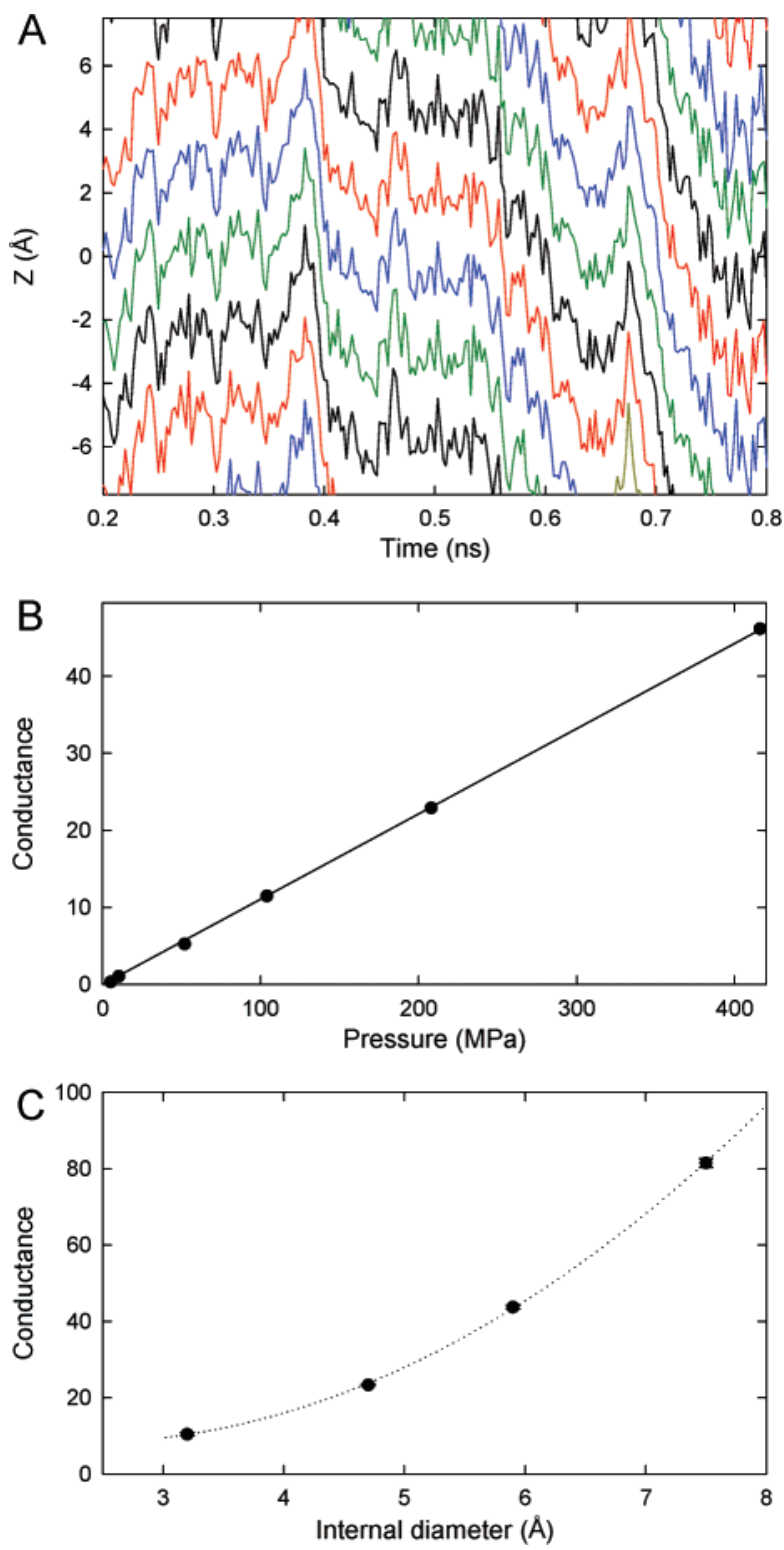

Figure 5. Water conductance through nanotubes. (A) Motions of the individual water molecules are plotted over a short section of the molecular dynamics simulation conducted with a hydrostatic pressure difference of $208 \mathrm{MPa}$ across the $(6,6)$ nanotube membrane. The conductance of the membrane (in water molecules per tube per ns) is plotted against (B) the applied hydrostatic pressure and (C) the internal diameter of the nanotube.

with the length of the tube as ions will find it harder to have enough sequential hops in the same direction to pass through longer pores as described elsewhere. ${ }^{50,51}$

There is also a simple relationship between the water conductances measured for each of the different sized tubes in this study, with the conductance being roughly proportional to the number of water chains that can form through the pore. Thus, the conductance of the $(7,7)$ and $(8,8)$ tubes are roughly double and quadruple that of the $(6,6)$ tube respectively. Although a single chain forms in both the $(5,5)$ and $(6,6)$ tubes, the conductance of the former is a little under half the latter due to the fact that water chains only form across the narrower pore half of the time (Figure 3).

As suspected from the PMFs, ions are seen to cross both the $(7,7)$ (2 ions in $25 \mathrm{~ns})$ and $(8,8)$ (28 ions in $17 \mathrm{~ns})$ membranes under a pressure differential, but none cross either of those formed from the narrower pores. Indeed a second PMF for the
TABLE 3: Desalination Potential of Carbon Nanotube Membranes $^{a}$

\begin{tabular}{|c|c|c|c|c|c|}
\hline \multirow[b]{2}{*}{ size } & \multirow[b]{2}{*}{$\begin{array}{c}\text { salt } \\
\text { rejection }\end{array}$} & \multicolumn{2}{|c|}{$\begin{array}{c}\text { maximal } \\
\text { pore density }\end{array}$} & \multicolumn{2}{|c|}{$\begin{array}{c}2.5 \times 10^{11} \\
\text { pores per cm }{ }^{2}\end{array}$} \\
\hline & & $\begin{array}{l}\text { flow } \\
\text { rate }\end{array}$ & improvement & $\begin{array}{l}\text { flow } \\
\text { rate }\end{array}$ & improvement \\
\hline$(5,5)$ & $100 \%$ & 45.2 & 682 & 0.16 & 2.42 \\
\hline$(6,6)$ & $100 \%$ & 78.8 & 1189 & 0.27 & 4.21 \\
\hline$(7,7)$ & $95 \%$ & 119.5 & 1801 & 0.42 & 6.39 \\
\hline$(8,8)$ & $58 \%$ & 182.9 & 2759 & 0.65 & 9.76 \\
\hline
\end{tabular}

${ }^{a}$ Numbers assume an operating pressure of $5.5 \mathrm{MPa}$ and allow for an osmotic pressure of $2.4 \mathrm{MPa}$. Flow rates are measured in $\mathrm{L} \mathrm{cm}^{-2}$ day $^{-1}$. Improvements in efficiency are quoted relative to published values for a FILMTEC SW30HR-380 commercial reverse osmosis membrane. ${ }^{57}$

$(6,6)$ membrane conducted with $208 \mathrm{MPa}$ of hydrostatic pressure indicates that the energy barrier of this pore to $\mathrm{Na}^{+}$is reduced by less than $1 \mathrm{kcal} / \mathrm{mol}$ by this large pressure differential, and ions can still not be expected to cross. Notably, $\mathrm{Na}^{+}$crosses these pores more readily than $\mathrm{Cl}^{-}$. No $\mathrm{Cl}^{-}$are seen to cross the $(7,7)$ membrane and five cross the $(8,8)$ membrane in $25 \mathrm{~ns}$ compared to $23 \mathrm{Na}^{+}$. This may be a consequence of the fact that the larger anion requires more water molecules to be removed from its solvation shell to enter these pores than does $\mathrm{Na}^{+}$. In these simulations the preferential conductance of $\mathrm{Na}^{+}$ compared to $\mathrm{Cl}^{-}$does not create a charge separation due to the periodic boundaries that join the reservoirs on either side of the membrane. In a real life situation, charging of the membrane created by preferential conduction of $\mathrm{Na}^{+}$can be avoided by circulating the seawater on the upstream side of the membrane.

Use for Desalination. To determine the suitability of CNTs for water desalination, we calculate the efficiency of salt rejection of each of our nanotube membranes (calculated from the number of water molecules and ions crossing the membrane during our simulations) and the flow rate of water through them as shown in Table 3. All of the tubes examined lead to some degree of salt rejection, dropping from $100 \%$ for the two narrower tubes to around $60 \%$ for the widest tube. Importantly this information determines how precisely the size of the nanotubes must be controlled if CNT membranes are to be fabricated for the purpose of desalination. Little desalination can be expected to occur if the tubes are greater in diameter than the $(8,8)$ pore studied here as there is unlikely to be a sizable barrier to ion permeation. However, further investigation of the salt rejection of wider nanotubes is warranted as a previous study found an energy barrier to $\mathrm{Na}{ }^{+}$conduction to be similar in a $(10,10)$ CNT to that reported here for $(8,8) .{ }^{37}$ As a rough guide, these results suggest that to achieve $>95 \%$ salt rejection as required to convert seawater into that fit for human consumption in a single pass requires that less than 1 in 100 of the tubes forming the membrane are above the diameter of $10 \AA$ (that of the $(7,7) \mathrm{CNT}$ ). Passing the water through multiple membranes would allow the use of wider pores (such as $(8,8)$ CNTs), however this would come at the energy cost of having to recreate the hydrostatic pressure difference across the subsequent membranes.

To compare the efficiency of these simulated CNT membranes with existing semipermeable membranes used for desalination via reverse osmosis, the flow rate through the membranes was calculated under standard operating conditions of 5.5 $\mathrm{MPa}$ allowing for the osmotic pressure differences of seawater and fresh water on either side of the membrane. These simulations suggest that very large flows can be expected in all the membranes studied. Indeed, a membrane comprising $(7,7)$ 
nanotubes could be expected to obtain $95 \%$ desalination with a flow rate of over 1500 times that of existing membranes. Lower pressures could be used in CNT membranes provided they overcome the osmotic pressure difference between seawater and fresh water $(2.4 \mathrm{MPa})$; thus, an examination of the trade off between flow rates and energy cost would be important in applying this technology.

The flow rate, of course, is highly dependent on the density of CNT pores in the membrane, and the results obtained here represent the maximum possible efficiency as the CNTs were packed directly beside each other with no intervening material. In reality, the fabrication of CNT membranes that are stable under large hydrostatic pressures is likely to require the CNTs to be packed within a solid nonporous material to prevent them coming apart, and thus the pore density can be expected to be significantly lower. In a recent study, CNTs are packed within a silicon nitride framework at a density of $2.5 \times 10^{11}$ pores per $\mathrm{cm}^{2}$, yielding some indication of the pore densities that can be achieved. ${ }^{18}$ Using this pore density and assuming $100 \%(7,7)$ nanotubes would yield an improvement in flow rate of 6 times that of existing technology. Improved fabrication techniques that achieved a higher density of pores within the membrane could be expected to increase this further.

\section{Conclusions}

Using molecular dynamics simulations, it is shown that membranes comprising sub nanometer diameter carbon nanotubes can provide an efficient means of water desalination when used in reverse osmosis. Although these narrow pores reject ions extremely well, they still conduct water at high rates and thus may be many times more efficient than existing membranes. A comparison of the properties of membranes formed by a range of differently sized tubes allows us to ascertain how precisely CNT membranes must be fabricated to achieve a desired level of desalination as well as yielding an estimate of the efficiency gains that could be achieved over existing technologies. Efficency gains of 5-1000 times could be expected if membranes comprising less than 1 pore in 100 over the size of $10 \AA$ in diameter can be made. Such membranes would have to be sufficiently strong to withstand the hydrostatic pressures applied to them, and how this could be achieved and the cost involved in fabrication has not been discussed here. However, it is encouraging that significant control over the size of CNTs incorporated into membranes has been obtained. ${ }^{18}$ Additional practical considerations that have not been discussed here include the possibility that organic solutes may partition into the nanotubes preventing water conduction ${ }^{52}$ as well as the potential fouling of these membranes by algaes and other contaminants. It is worth noting that the primary causes of salt rejection and water transport in these studies is the narrow, smooth, nonpolar nature of the CNTs and thus the filtration seen here should not be specific to the type of CNT used provided the pore has the correct dimensions. That is, tubes of different chirality as well as double or multiwalled tubes should produce similar results.

A detailed examination of the properties of ion and water transport that make these nanotubes so efficient for desalination has also been presented. The energy barriers arise predominantly at the entrance and exit of the tubes meaning that transport is almost frictionless once molecules are inside. The permeation of ions into the nonpolar interior of the CNTs is largely controlled by the energy required to remove the hydrating water molecules. Although hydrogen bonds must be broken for water to enter these pores, the stability of the remaining hydrogen bonds in the pore compared to those in the bulk means that there is no energy cost for entry. These results highlight the remarkable conclusion that very efficient transport of water molecules is possible in narrow nonpolar pores.

In addition to designing membranes for desalination, these studies are also useful for understanding transport in biological water and ion channels. The rapid single file water diffusion seen in the narrow CNTs resembles that in aquaporin channels, and the wetting/dewetting transitions in the $(5,5)$ pore are similar to those observed in mechanosensitive ion channels and the nictotinic acetylcholine receptor. ${ }^{25,27}$ In particular, this study supports previous work suggesting that narrow nonpolar pores provide a very efficient mechanism of gating ionic currents. ${ }^{24,27,37}$ Although ions do not pass through the nanotubes in our simulations when the effective internal diameter is less than $\sim 5 \AA$, rapid transport arises when this is increased only slightly to $7.5 \AA$, a similar result to that obtained for the nicotinic receptor. ${ }^{27}$ This means that gated ion channels incorporating hydrophobically lined regions can move between open and closed states with only minimal conformational changes. Such hydrophobically lined regions of biological ion channels appear to be common, being present for example in potassium channels, ${ }^{53,54}$ mechanosensitive channels, ${ }^{55}$ and the nicotinic acetylcholine receptor. ${ }^{56}$

Although desalination is widely used throughout the world, the development of more efficient membranes that can separate salt from water while maintaining large throughput has the potential to make such desalination cheaper and fresh water more widely available. Here we show that porous membranes incorporating narrow carbon nanotubes can in principle achieve water desalination with flow rates far exceeding existing technologies.

Acknowledgment. This work was supported by an award under the Merit Allocation Scheme on the National Facility of the Australian Partnership for Advanced Computing and additional computer time from the iVEC high performance computing facility. Dr Corry is supported by funding from the Australian Research Council and the Australian National Health and Medical Research Council.

\section{References and Notes}

(1) Fourth Assessment report of the Intergovernmental Panel on Climate Change; Soloman, S.; qin, D.; Manning, M.; Chen, Z.; Marquis, M.; Averyt, K.; Tignor, M.; Miller, H. Eds. Cambridge Uni Press, Cambridge, UK, 2007; Chapter Climate Change 2007: the physical science basis.

(2) Zeidel, M.; Ambudkar, S.; Smith, B.; Agre, P. Biochemistry 1992, $31,7436-7440$

(3) Agre, P.; King, L.; Yasui, M.; Guggino, W.; Ottersen, O. J. Physiol. 2001, 552, 3-16.

(4) Sui, H.; Han, B.; Lee, J.; Walian, P.; Jap, B. Nature 2001, 414, $872-878$.

(5) Iijima, S. Nature 1991, 354, 56-58.

(6) Dresselhaus, M.; Dresselhaus, G.; eds, P. A. Carbon nanotubes: synthesis, structure, properties and applications; Springer, Heidelberg, 2001.

(7) Mitchell, D.; Lee, S.; Trofin, L.; Nevanen, T.; Soderlund, H.; Martin, C. J. Am. Chem. Soc. 2002, 124, 11864-11865.

(8) Carbon nanotubes: structure and applications; Meyyappan, M., Ed.; CRC Press: Boca Raton, FL, 2005.

(9) Zhang, D.; Shi, L.; Fang, J.; Dai, K.; Liu, J. Mater. Chem. Phys. 2006, 96, 140-144.

(10) Zhang, D.; Shi, L.; Fang, J.; Dai, K. J. Mater. Sci. 2007, 42, 24712475

(11) Li, J.; Papadopoulos, C.; Xu, J. M.; Moskovits, M. Appl. Phys. Lett. 1999, 75, 367-369.

(12) Hata, K.; Futaba, D. N.; Mizuno, K.; Namai, T.; Yumura, M.; Iijima, S. Science 2004, 306, 1362-1364.

(13) Talapatra, S.; Kar, S.; Pal, S. K.; Vajtai, R.; Ci, L.; Victor, P.; Shaijumon, M. M.; Kaur, S.; Nalamasu, O.; Ajayan, P. M. Nat. Nanotechnol. 2006, 1, 112-116. 
(14) Yamada, T.; Namai, T.; Hata, K.; Futaba, D. N.; Mizuno, K.; Fan, J.; Yudasaka, M.; Yumura, M.; Iijima, S. Nat. Nanotechnol. 2006, 1, 131136.

(15) Miller, S. A.; Young, V. Y.; Martin, C. R. J. Am. Chem. Soc. 2001, $123,12335-12342$.

(16) Hinds, B. J.; Chopra, N.; Rantell, T.; Andrews, R.; Gavalas, V.; Bachas, L. G. Science 2003, 303, 62-65.

(17) Holt, J. K.; Noy, A.; Huser, T.; Eaglesham, D.; Bakajin, O. Nano Lett. 2004, 2, 2245-2250.

(18) Holt, J.; Park, H; Wang, Y; Stadermann, M; Artyukhin, A. Grigoropolous, C.; Noy, A.; Bakajin, O. Science 2006, 312, 1034-1037.

(19) Mao, Z.; Sinnott, S. B. J. Phys. Chem. B 2001, 105, 6916-6924.

(20) Hummer, G.; Rasiah, J.; Nowortya, J. Nature 2001, 414, 188190.

(21) Kalra, A.; Garde, S.; Hummer, G. Proc. Natl. Acad. Sci. U.S.A. 2003, 100, 10175-10180.

(22) Striolo, A. Nano Lett. 2006, 6, 633-639.

(23) Beckstein, O.; Sansom, M. Proc. Natl. Acad. Sci. U.S.A. 2003, 100 , 7063-7068.

(24) Beckstein, O.; Sansom, M. Phys. Biol. 2004, 1, 42-52.

(25) Anishkin, A.; Sukharev, S. Biophys. J. 2004, 86, 2883-2895.

(26) Sotomayor, M.; Schulten, K. Biophys. J. 2004, 87, 3050-3065.

(27) Corry, B. Biophys. J. 2006, 90, 799-810.

(28) Beckstein, O.; Sansom, M. Phys. Biol. 2006, 3, 147-159.

(29) Waghe, A.; Rasaiah, J.; Hummer, G. J. Chem. Phys. 2002, 117, 10789-10795.

(30) Mann, D.; Halls, M. Phys. Rev. Lett. 2003, 90, 195503-195506.

(31) Dellago, C.; Naor, M.; Hummer, G. Phys. Rev. Lett. 2003, 90, 105902-105905.

(32) Zhu, F.; Schulten, K. Biophys. J. 2003, 85, 236-244. 805 .

(33) Koga, K.; Gao, G.; Tanaka, H.; Zeng, X. Nature 2001, 412, 802-

(34) Noon, W.; Ausman, K.; Smalley, R.; Ma, J. Chem. Phys. Lett. 2002, $355,445-448$.

(35) Huang, B.; Xia, Y.; Zhao, M.; Liu, F. L.; Ji, Y.; Song, C. J. Chem. Phys. 2005, 122, 084708 .

(36) Hanasaki, I.; Nakatani, A. J. Chem. Phys. 2006, 124, 174714174722 .
(37) Peter, C.; Hummer, G. Biophys. J. 2005, 89, 2222-2234.

(38) Liu, H.; Murad, S.; Jameson, C. J. J. Chem. Phys. 2006, 125 084713.

(39) Lu, D.; Li, Y.; Ravaioli, U.; Schulten, K. J. Phys. Chem. B 2005, $109,11461-11467$

(40) Torrie, G.; Valleau, J. Chem. Phys. Lett. 1974, 28, 578-581.

(41) Kumar, S.; Bouzida, D.; Swendsen, R.; Kollman, P.; Rosenberg, J. J. Comput. Chem. 1992, 13, 1011-1021.

(42) Roux, B. Comput. Phys. Commun. 1995, 91, 275-282.

(43) Zhu, F.; Tajkhorshid, E.; Schulten, K. Biophys. J. 2002, 83, 154160.

44) Zhu, F.; Tajkhorshid, E.; Schulten, K. Biophys. J. 2004, 86, 5057.

(45) Phillips, J.; Braun, R.; Wang, W.; Gumbart, J.; Tajkhorshid, E.; Villa, E.; Chipot, C.; Skeel, R.; Kale, L.; Schulten, K. J. Comput. Chem. 2005, 26, 1781-1802.

(46) MacKerell, A. D., Jr.; et al. J. Phys. Chem. B 1998, 102, 35863616.

(47) Dzubiella, J.; Allen, R.; Hansen, J. J. Chem. Phys. 2004, 120, 50015004.

(48) Loof, H. D.; Nilsson, L.; Rigler, R. J. Am. Chem. Soc. 1992, 114, 4028-4035.

(49) Skoulidas, A. I.; Ackerman, D. M.; Johnson, J. K.; Sholl, D. S. Phys. Rev. Lett. 2002, 89, 185901-185904.

(50) Berezhkovskii, A.; Hummer, G. Phys. Rev. Lett. 2002, 89, 064503.

(51) Li, J.; Yang, Z.; Fang, H.; Zhou, R.; Tang, X. Chin. Phys. Lett. 2007, 24, 2710-2713.

(52) Kalra, A.; Hummer, G.; Garde, S. J. Phys. Chem. B 2004, 108, 544-549.

(53) Doyle, D. A.; Cabral, J. M.; Pfuetzner, R. A.; Kuo, A.; Gulbis, J. M.; Cohen, S. L.; Chait, B. T.; MacKinnon, R. Science 1998, 280, 69-77.

(54) Jiang, Y.; Lee, A.; Chen, J.; Cadene, M.; Chait, B.; MacKinnon, R. Nature 2002, 417, 523-526.

(55) Bass, R.; Strop, P.; Barclay, M.; Rees, D. Science 2002, 298, 15821587.

(56) Miyazawa, A. Y. F.; Unwin, N. Nature 2003, 423, 949-955.

(57) http://www.dow.com/liquidseps/prod/sw30hr_380.htm,. 